\title{
Ultrasonic Properties of Plutonium Monochalcogenides
}

\author{
Devraj Singh $^{1}$, Vyoma Bhalla ${ }^{1, *}$ Sudhanshu Tripathi' ${ }^{2}, V_{\text {inod Kumar Singh }}^{3}$, A.K.Gupta ${ }^{4}$ \\ ${ }^{1}$ Department of Applied Physics, Amity School of Engineering and Technology, Bijwasan, New Delhi-110061, India \\ ${ }^{2}$ Department of Instrumentation \& Control Engineering, Amity School of Engineering and Technology, New Delhi-110061, India \\ ${ }^{3}$ Department of Physics, V.S.S.D. College, Kanpur-208002, India \\ ${ }^{4}$ Academic Department, National Institute of Open Schooling, NOIDA-201309, India \\ *Corresponding Author: vyoma05@yahoo.co.in
}

Copyright @ 2014 Horizon Research Publishing All rights reserved.

\begin{abstract}
A computer program in MATLAB is developed for the numerical computation of ultrasonic properties in $\mathrm{Pu}$ - monochalcogenides in present investigation in the temperature range $100-300 \mathrm{~K}$ along $\langle 100\rangle,<110\rangle$ and $111>$ directions. The program calculates the second and third order elastic constants, relaxation time, ultrasonic coupling constants and ultrasonic attenuation by considering the interaction of sound wave with complete spectrum of thermal phonon modes and by taking two basic parameters i.e., nearest neighbour distance and hardness parameters. The program has been successful for the study of (i) temperature dependence of the second and third order elastic constants, (ii) temperature and crystallographic dependence of ultrasonic velocities for longitudinal and shear waves with thermal relaxation time, ultrasonic coupling constants and ultrasonic attenuation for PuS, PuSe and PuTe. The behaviour of acoustic parameters as a function of higher temperature has been discussed in correlation with other thermophysical parameters.
\end{abstract}

Keywords Plutonium Monochalcogenides, Elastic Properties, Ultrasonic Properties

\section{Introduction}

Almost all the monochalcogenides, both the lanthanides as well as actinides crystallize in the cubic $\mathrm{NaCl}$-type structure. Their transport properties can be very complex. The plutonium monochalcogenides display a variety of anomalous properties and is the topic of research for more than 50 years [1-10]. The ultrasonic and elastic properties are the versatile tools to characterize the material behavior. These properties are well connected to thermal conductivity, specific heat, heat capacity, pressure and electrical resistivity. Petit et al. studied the electronic and magnetic properties of plutonium monochalcogenides using ab-initio selfinteraction corrected (SIC) local spin density (LSD) approximation [1]. The semimetallic nature of plutonium monochalcogenides have been proved by Oppeneer et al. on the basis of electronic structure calculation [2]. Wachter experimentally verified the intermediate valence nature of plutonium monochalcogenides [3]. Magnetic properties of plutonium monochalcogenides have been discussed using LDA+U calculation by Havela et al. [4]. Suzuki and Oppeneer discussed the electronic structure and Kondo insulator state of the $\mathrm{Pu}$ chalcogenides using the LDA, the LDA $+\mathrm{U}$ and LDA + DMFT methods of computation [5]. Mattenberger and Vogt investigated magnetic properties of $\mathrm{Pu}$ monochalcogenides at different temperature [6]. The electronic structures were computed for plutonium monochalcogenides using a relativistic APW and the local density approximation by Hasegawa and Yamagami [7]. Fournier et al. reported resistivity measurements on single crystals of PuS, PuSe and PuTe. They found that the resistivity of all three monochalcogenides increased continuously from room temperature down to lowest temperature. They suggested them as small gap semiconductor [8]. Wachter et al. [9] showed that the plutonium monochalcogenides represent the high pressure phase of samarium monochalcogenides i.e., the intermediate valent state. A very direct experiment to prove intermediate valence to occur, a negative $C_{12}$ is being typical for this phenomenon for PuTe was given by Mendik et al. [10].

In the present paper, the temperature dependence of ultrasonic attenuation and other related parameters have been studied using a computer program in MATLAB, showing some characteristic features for the chosen monochalcogenides along $<100>,<110>$ and $<111>$ crystallographic directions. At high temperature $(>100 \mathrm{~K})$, phonon-phonon interaction is the dominant cause of the ultrasonic attenuation in all type of solids. In metals, due to hogher thermal conductivity, a part of total ultrasonic attenuation arises due to thermo-elastic loss. The secondand third-order elastic constants (SOECs and TOECs) have been evaluated at different temperatures using Coulomb and Born-Mayer potential and two basic parameters i.e. nearest neighbour distance and hardness parameter as input.

\section{Theory}


Mason theory [11] for solids, which was initially proposed by Akhieser [12] has been established for the temperature dependence of ultrasonic attenuation and other related parameter for different types of advanced materials along different propagation directions of the wave. This theory is still to be established for the variety of several types of advanced materials along different crystallographic orientations. The effort has been made to establish this theory to evaluate ultrasonic absorption coefficients and related parameters of $\mathrm{PuX}(\mathrm{X}: \mathrm{S}, \mathrm{Se}, \mathrm{Te})$ at $100-300 \mathrm{~K}$ along $<100>,<110>$ and $<111>$ orientations. Formulation for ultrasonic parameters $\left(\omega \tau_{\mathrm{th}}<<1\right)$ is given by:

$$
\begin{gathered}
\alpha_{A k h}=\frac{\omega^{2} E_{0} D n \tau_{t h}}{6 \rho V^{5}} \\
\alpha_{t h}=\frac{\omega^{2}\left\langle\gamma_{j}^{i}\right\rangle K T}{2 \rho V^{5}} \\
\tau_{t h}=\frac{1}{2} \tau_{l}=\tau_{s}=\frac{3 K}{C_{V} \overline{V^{5}}}
\end{gathered}
$$

where

$$
\begin{gathered}
\frac{3}{\bar{V}^{3}}=\frac{1}{V_{l}^{3}}+\frac{3}{V_{S}^{3}} \\
D=9\left\langle\left(\gamma_{i}^{j}\right)^{2}\right\rangle-3 \frac{\left\langle\left(\gamma_{i}^{j}\right)^{2}\right\rangle C_{V} T}{E_{0}}
\end{gathered}
$$

$\alpha_{A k h}$ is the ultrasonic absorption coefficient due to phonon- phonon interaction mechanism which is valid at higher temperatures, $\alpha_{t h}$ is the thermoelastic loss, $\omega$ is angular frequency, D is non-linearity parameter, $\left\langle\gamma_{i}^{j}\right\rangle$ is average Grüneisen numbers. Grüneisen parameters are the ratios of SOECs and TOECs. These parameters were evaluated using Mason's table given in literature [11]. The value of $\mathrm{n}$ is equal to 1 for shear wave and 2 for longitudinal wave, $\tau_{\text {th }}$ thermal relaxation time, $\mathrm{V}$ is the velocity of wave, $\rho$ is the density of the material, $\mathrm{Cv}$ is specific heat per unit volume, E0 is the internal energy density, $\mathrm{T}$ is the temperature in Kelvin scale and 'l', 's' represents longitudinal and shear respectively.

For the evaluation of ultrasonic properties we have also evaluated temperature dependent SOECs and TOECs following Brugger's definition of elastic constants $[13,14]$.The obtained values of SOECs and TOECs have been used to find out the values of various thermal properties of the materials. Flow diagram for complete evaluation is given below in Figure 1.

\section{Results and Discussion}

The computed values of SOECs and TOECs using two basic parameters viz nearest neighbor distance, $r_{0}$ and hardness parameter, $b$ are presented in Table 1 . The thermal relaxation time and acoustic coupling constant for longitudinal and shear mode of wave propagation along $<100>,<110>$ and $<111>$ directions is computed using Eqs.(3)-(4) and presented in Table 2. Finally, calculated values of temperature and orientation dependent ultrasonic attenuation computed with the help of Eqs.(1)-(2) are presented in Table 3. The flow chart of computational steps of the present investigation is as given below in Figure 2.

The mechanical strength and durability of the material are related to their elastic constants. Table 1 depicts that $\mathrm{C}_{111}$, $\mathrm{C}_{112}$ and $\mathrm{C}_{116}$ are negative valued TOEC while other TOECs and SOECs are positive. It is obvious from Table 1 that $\mathrm{C}_{11}$, $\mathrm{C}_{44}, \mathrm{C}_{112}, \mathrm{C}_{144}, \mathrm{C}_{166}$ are increasing with increase in temperature whereas $\mathrm{C}_{12}, \mathrm{C}_{111}, \mathrm{C}_{123}$ decreases with increase in temperature, while $\mathrm{C}_{456}$ remains constant at every temperature due to zero vibrational contribution. If inter-atomic distance increases or decreases with temperature then interaction potential decreases or increases, which causes decrease or increase in elastic constants. This type of behaviour has been found already in other $B_{1}$ structured materials [15-18]. The variation of $D_{1} / D_{S}$ with temperature in Table 2 indicates that acoustic coupling constant decreases with temperature for wave propagating along $<100\rangle,<110\rangle$ and $<111>$ directions and polarized along $<100>,<110>$ and $<\overline{1} 10>$ directions respectively, while for wave propagating along $<110>$ direction with polarization along $<001>$, the $D_{1} / D_{S}$ are found to increase with temperature. The acoustic coupling constant is the measure of acoustic energy converted to thermal energy under the thermal relaxation process. Thus, the decrease in $\mathrm{D}_{1} / \mathrm{D}_{\mathrm{S}}$ with temperature shows the decrease in longitudinal loss with temperature or vice versa [18]. A comparison for D has been made with similar type $\mathrm{B}_{1}$ structured material i.e., with $\mathrm{LiF}$ in Table 2 [19]. It can be seen from Table 2 that the order of thermal relaxation, $\tau_{\text {th }}$ for PuX is $10^{-13} \mathrm{~s}-10^{-14} \mathrm{~s}$. On the basis of $\tau_{\text {th }}$, one may predict the materials to be semiconductors $[19,20]$.

It is clear from Table 3 that ultrasonic attenuation due to thermoelastic relaxation mechanism i.e., thermoelastic loss $\left(\alpha / \mathrm{f}^{2}\right)_{\text {th }}$ is negligible in comparision to total attenuation, $\left[\left(\alpha / f^{2}\right)_{\text {total }}=\left(\alpha / f^{2}\right)_{\text {long }}+\left(\alpha / f^{2}\right)_{\text {shear }}+\left(\alpha / f^{2}\right)_{\text {total }}\right]$

Total attenuation increases with increase in temperature. This type of behavior is similar to previous studied materials $[15-18,20,21]$. The order of attenuation is the same as previous studied semiconducting materials [20,21].

Here it is noticeable that the completed ultrasonic attenuation is lowest for PuS along all the directions. It is also obvious that the value of ultrasonic attenuation along $<100>$ is lowest. So we may say that PuS is more useful along $<100>$ direction. 


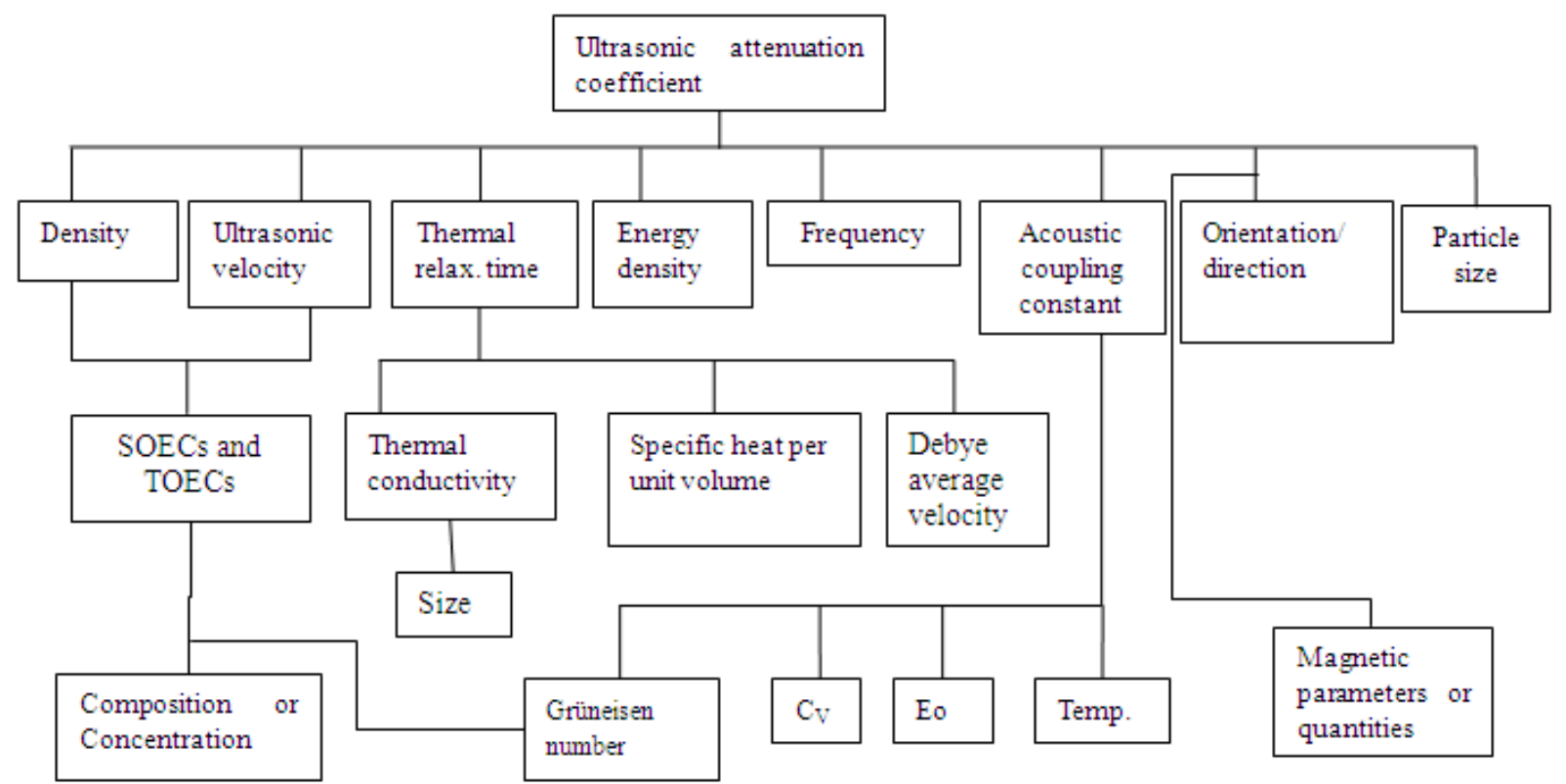

Figure 1. Complete evaluation of ultrasonic attenuation

Table 1. Temperature dependent SOECs and TOECs of PuX ( in $10^{10} \mathrm{~N} / \mathrm{m}^{2}$ ).

\begin{tabular}{|c|c|c|c|c|c|c|c|c|c|c|}
\hline Material & Temp $[\mathrm{K}]$ & $\mathrm{C}_{11}$ & $\mathrm{C}_{12}$ & $\mathrm{C}_{44}$ & $\mathrm{C}_{111}$ & $\mathrm{C}_{112}$ & $\mathrm{C}_{123}$ & $\mathrm{C}_{144}$ & $\mathrm{C}_{166}$ & $\mathrm{C}_{456}$ \\
\hline \multirow{3}{*}{$\mathrm{PuS}$} & 100 & 5.841 & 1.417 & 1.515 & -96.52 & -5.749 & 2.005 & 2.565 & -6.156 & 2.546 \\
\hline & 200 & 6.029 & 1.327 & 1.518 & -96.86 & -5.402 & 1.453 & 2.583 & -5.402 & 2.546 \\
\hline & 300 & 6.171 & 1.242 & 1.525 & -96.85 & -5.074 & 0.925 & 2.602 & -6.198 & 2.546 \\
\hline \multirow{3}{*}{$\mathrm{PuSe}$} & 100 & 5.145 & 1.201 & 1.289 & -85.28 & -4.860 & 1.671 & 2.199 & -5.221 & 2.182 \\
\hline & 200 & 5.306 & 1.119 & 1.293 & -85.98 & -4.536 & 1.159 & 2.215 & -5.240 & 2.182 \\
\hline & 300 & 5.486 & 1.037 & 1.298 & -86.88 & -4.207 & 0.647 & 2.231 & -5.262 & 2.182 \\
\hline \multirow{3}{*}{ PuTe } & 100 & 4.178 & 0.961 & 0.992 & -70.21 & -3.688 & 0.992 & 1.707 & -4.003 & 1.695 \\
\hline & 200 & 4.326 & 0.844 & 0.995 & -70.92 & -3.396 & 0.995 & 1.721 & -4.019 & 1.695 \\
\hline & 300 & 4.746 & 0.748 & 0.988 & -77.23 & -2.956 & 0.177 & 1.733 & -3.974 & 1.695 \\
\hline
\end{tabular}

Table 2. Temperature dependent $\mathrm{D}$ and $\tau_{\text {th }}\left(\right.$ in $\left.10^{-12} \mathrm{~s}\right)$ of $\mathrm{PuX}$.

\begin{tabular}{|c|c|c|c|c|c|c|c|c|c|}
\hline Material & Temp[K] & $\mathrm{D}_{\mathrm{l}}$ & $\mathrm{D}_{\mathrm{S}}$ & $\mathrm{D}_{1}$ & $\mathrm{D}_{\mathrm{S} 1}$ & $\mathrm{D}_{\mathrm{S} 2}$ & $\mathrm{D}_{\mathrm{l}}$ & $\mathrm{D}_{\mathrm{s}}$ & $\tau_{\text {th }}$ \\
\hline $\begin{array}{l}\text { Direction of } \\
\text { Propagation }\end{array}$ & - & $<100>$ & $<100>$ & $<110>$ & $<110>$ & $<110>$ & $<111>$ & $<111>$ & - \\
\hline $\begin{array}{l}\text { Direction of } \\
\text { Polarization } \\
\end{array}$ & - & - & $<100>$ & - & $<001>$ & $<1 \overline{\mathbf{1}} 0>$ & - & $<\overline{1} 10>$ & - \\
\hline \multirow{3}{*}{$\mathrm{PuS}$} & 100 & 18.249 & 1.220 & 17.809 & 2.565 & 28.957 & 15.146 & 19.56 & 0.01 \\
\hline & 200 & 17.671 & 1.206 & 16.973 & 2.242 & 28.031 & 14.404 & 19.56 & 0.02 \\
\hline & 300 & 16.782 & 1.197 & 15.728 & 2.043 & 26.775 & 13.393 & 18.95 & 0.05 \\
\hline \multirow{3}{*}{$\mathrm{PuSe}$} & 100 & 19.435 & 1.201 & 18.282 & 2.303 & 29.039 & 15.519 & 18.13 & 0.03 \\
\hline & 200 & 18.845 & 1.212 & 18.248 & 2.039 & 28.708 & 14.551 & 20.24 & 0.06 \\
\hline & 300 & 18.025 & 1.199 & 15.832 & 1.818 & 27.513 & 13.458 & 19.42 & 0.14 \\
\hline \multirow{3}{*}{ PuTe } & 100 & 18.355 & 1.187 & 18.602 & 1.187 & 31.096 & 15.775 & 18.63 & 0.09 \\
\hline & 200 & 17.116 & 1.188 & 17.035 & 1.741 & 29.691 & 14.470 & 21.02 & 0.10 \\
\hline & 300 & 16.225 & 1.161 & 16.422 & 1.332 & 29.844 & 13.818 & 20.10 & 0.54 \\
\hline \multirow{2}{*}{$\operatorname{LiF}[19]$} & $300^{\text {Theor }}$ & 38 & 3 & 15 & 12 & 18 & 18 & - & - \\
\hline & $300^{\text {Exp }}$ & 38 & 45 & 17 & 4.5 & 35 & 11 & 21 & - \\
\hline
\end{tabular}




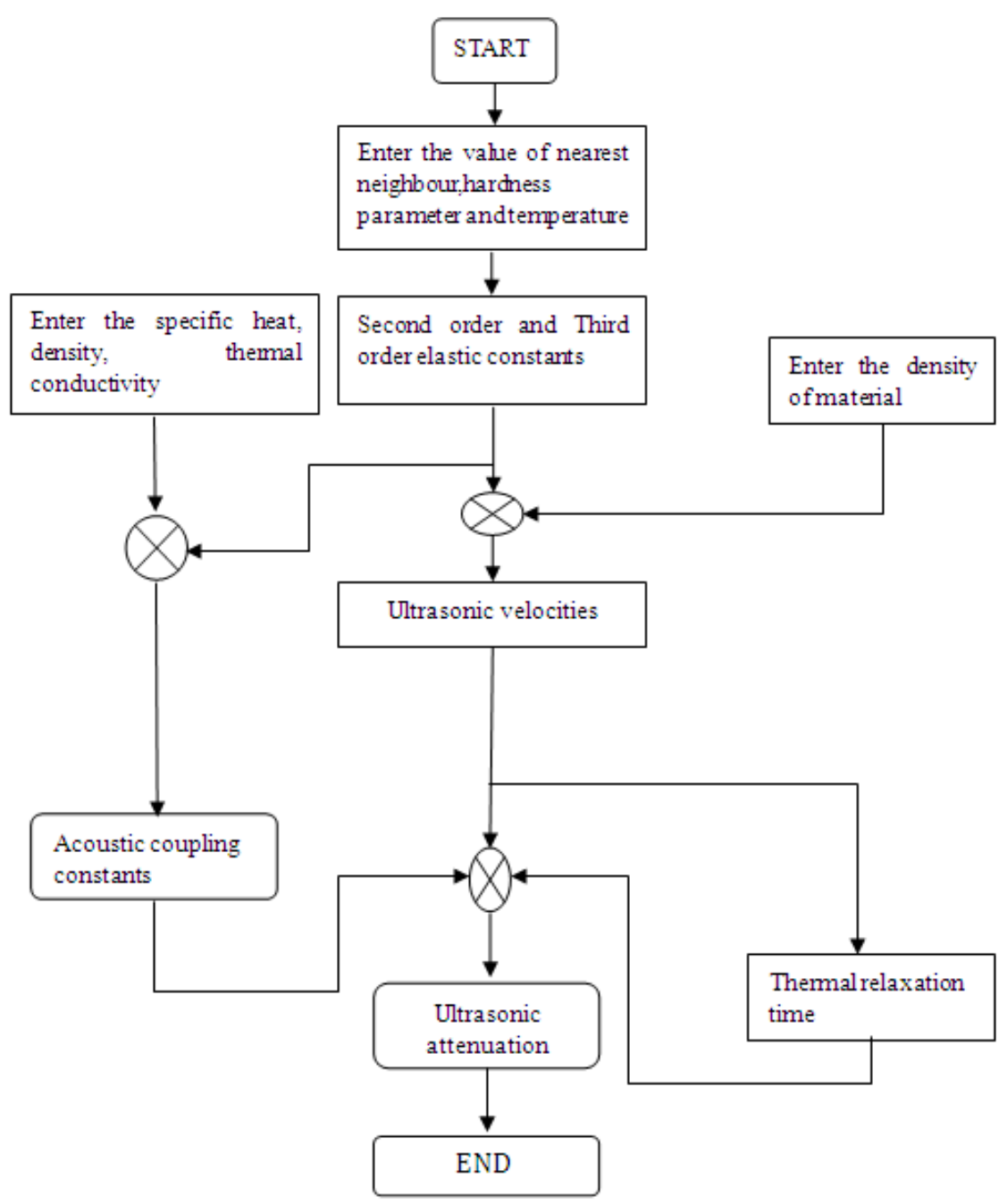

Figure 2. Computational concepts for the determination of Ultrasonic attenuation

Table 2. Temperature dependent $\mathrm{D}$ and $\tau_{\text {th }}$ ( in $10^{-12} \mathrm{~s}$ ) of PuX.

\begin{tabular}{|c|c|c|c|c|c|c|c|c|c|}
\hline Material & Temp[K] & $\mathrm{D}_{1}$ & $\mathrm{D}_{\mathrm{S}}$ & $\mathrm{D}_{1}$ & $\mathrm{D}_{\mathrm{S} 1}$ & $\mathrm{D}_{\mathrm{S} 2}$ & $\mathrm{D}_{1}$ & $\mathrm{D}_{\mathrm{S}}$ & $\tau_{\text {th }}$ \\
\hline $\begin{array}{l}\text { Direction of } \\
\text { Propagation }\end{array}$ & - & $<100>$ & $<100>$ & $<110>$ & $<110>$ & $<110>$ & $<111>$ & $<111>$ & - \\
\hline $\begin{array}{l}\text { Direction of } \\
\text { Polarization } \\
\end{array}$ & - & - & $<100>$ & - & $<001>$ & $<1 \overline{\mathbf{1}} 0>$ & - & $<\overline{\mathbf{1}} 10>$ & - \\
\hline \multirow{3}{*}{$\mathrm{PuS}$} & 100 & 18.249 & 1.220 & 17.809 & 2.565 & 28.957 & 15.146 & 19.56 & 0.01 \\
\hline & 200 & 17.671 & 1.206 & 16.973 & 2.242 & 28.031 & 14.404 & 19.56 & 0.02 \\
\hline & 300 & 16.782 & 1.197 & 15.728 & 2.043 & 26.775 & 13.393 & 18.95 & 0.05 \\
\hline \multirow{3}{*}{ PuSe } & 100 & 19.435 & 1.201 & 18.282 & 2.303 & 29.039 & 15.519 & 18.13 & 0.03 \\
\hline & 200 & 18.845 & 1.212 & 18.248 & 2.039 & 28.708 & 14.551 & 20.24 & 0.06 \\
\hline & 300 & 18.025 & 1.199 & 15.832 & 1.818 & 27.513 & 13.458 & 19.42 & 0.14 \\
\hline \multirow{3}{*}{ PuTe } & 100 & 18.355 & 1.187 & 18.602 & 1.187 & 31.096 & 15.775 & 18.63 & 0.09 \\
\hline & 200 & 17.116 & 1.188 & 17.035 & 1.741 & 29.691 & 14.470 & 21.02 & 0.10 \\
\hline & 300 & 16.225 & 1.161 & 16.422 & 1.332 & 29.844 & 13.818 & 20.10 & 0.54 \\
\hline \multirow{2}{*}{$\operatorname{LiF}[19]$} & $300^{\text {Theor }}$ & 38 & 3 & 15 & 12 & 18 & 18 & - & - \\
\hline & $300^{\operatorname{Exp}}$ & 38 & 45 & 17 & 4.5 & 35 & 11 & 21 & - \\
\hline
\end{tabular}


Table 3. Temperature dependent ultrasonic attenuation of PuX along different directions ( in $10^{-16} \mathrm{Nps}^{2} / \mathrm{m}$ ).

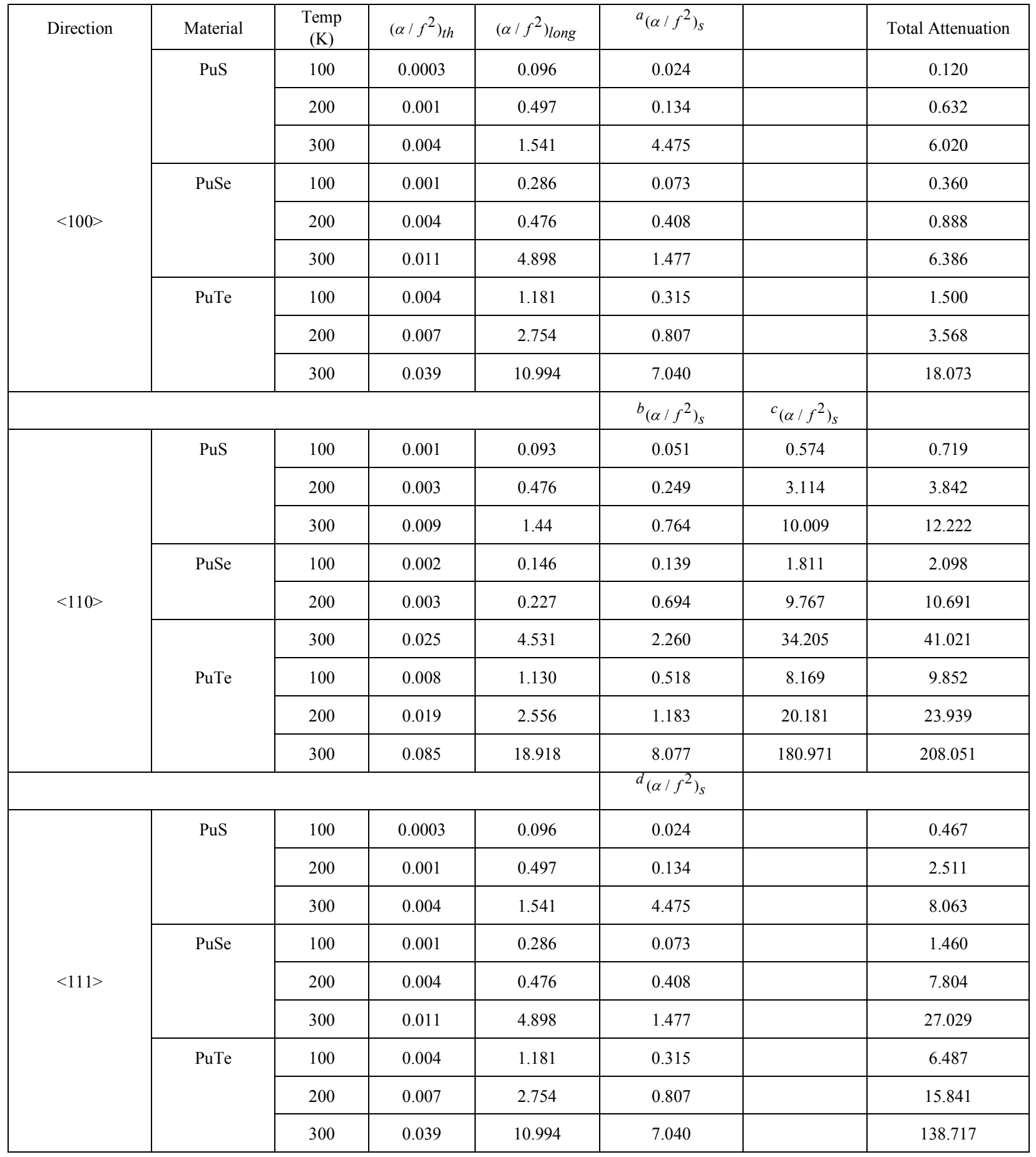

${ }^{a}$ shear wave polarized along $<100>$ cshear wave polarized along $<1 \overline{1} 0>$

${ }^{\mathrm{b}}$ shear wave polarized along $<001>$ dshear wave polarized along $<110>$

\section{Conclusion}

The following conclusions can be drawn on the basis of above discussion:

- We applied Coulomb and Born-Mayer potential to find out SOECs and TOECs. SOECs and TOECs have been compared with other structured materials, which are near to agreement, hence our approach to compute SOEC and TOEC appear to be justified.
- The thermal relaxation time of these materials has order of $10^{-13}$, which is the confirmation for semi-conducting materials.

- PuS is more useful in comparison to PuSe and PuTe on the basis of obtained ultrasonic attenuation.

- $<100>$ direction is more appropriate candidate for the wave propagation through these materials.

- The variation of ultrasonic attenuation clearly supports the present theoretical approach for ultrasonic attenuation, 
which is directly connected with the values of SOEC and TOEC based on some parameters.

Obtained results of present investigation for these semiconducting materials with a computer program in MATLAB may be useful to find theoretical and experimental finding of many physical properties like thermal conductivity, electrical sensitivity, infrared lattice resonance frequency, specific heat and various transport properties. These results can be used for engineering and technological applications.

\section{REFERENCES}

[1] L. Petit, A. Svane, W. M. Temmerman, Z. Szotek, Electronic structure of $\mathrm{Pu}$ monochalcogenides and monopnictides, European Journal of Physics B, Vol.25, No. 2, 139-146, 2002.

[2] P. M. Oppeneer, T. Kraft, M. S. S. Brooks, Electronic structure of plutonium monochalcogenides, Physical Review B, Vol.61, No.19, 12825-12834, 2001.

[3] P. Wachter, Intermediate valence of plutonium chalcogenides as deterned by photo emission, Solid State Communications, Vol.127, No.9, 599-603, 2003.

[4] L. Havela, A. Shick, T. Gouder, Magnetic properties of plutonium and $\mathrm{Pu}$ compounds, Journal of Applied Physics, Vol.105, No.1, 07E130-(1-3), 2009.

[5] M. Suzuki, P. M. Oppeneer, Theory of the insulator ground state of the plutonium monochalcogenides: AnLDA+DMFT investigation, IOP Conference Series: Material Science Engineering, Vol.9, No.1, 012086 (1-7), 2010.

[6] K. Mattenberger, O. Vogt, Magnetism of Monochalcogenides and Monopnictides of the Actinides, Physics Scripta, Vol.T45, 103-109, 1992

[7] A. Hasegawa, H. Yamagami, Electronic structure of plutonium monochalcogenides, Journal of Magnetism and Magnetic Materials, Vol.104-107, Part1, 65-66, 1992.

[8] J. M. Fournier, E. Pleska, J. Chiapusio, J. Rossat-Mignod, J. Rebizant, J. C. Spirlet, O. Vogt, Electrical resistivity of Plutonium monochalcogenides, Physica B, Vol.163, No.1-3, 493-495, 1990.
[9] P. Wacher, F. Marabelli, B. Bucher, Plutonium chalcogenides: Intermediate valence and electronic structure, Physical Review B, Vol.43, No.13, 11136-11144, 1991

[10] M. Mendik, P. Watcher, J. C. Sparlet, J. Rebizant, Intermediate valent PuTe: negative elastic constants, Physica B, Vol. 186-188, 678-680, 1993

[11] W. P. Mason, Physics Acoustics, Vol III B Academic Press, New York, 1965.

[12] A. Akhieser, On the absorption of sound in solids, Journal of Physics (Moscow), Vol.1, 277-287, 1939.

[13] K. Brugger, Thermodynamic definition of higher order elastic constants of the alkali halides crystals, Journal of Physical Society of Japan, Vol. 45, 1449-1456, 1978.

[14] S. Mori, Y. Hiki, Calculation of the third-and fourth-order elastic constants of the alkali halides crystals, Journal of Physical Society of Japan, Vol.45, 1449-1456, 1975.

[15] R. Kumar, D. Singh, S. Tripathi, Crystal Anharmonicity in strontium monochalcogenides, Asian Journal of Chemistry, Vol.24, No.12, 5652-5654, 2012.

[16] D. Singh, D. K. Pandey, D. K. Singh, R. R. Yadav, Propagation of ultrasonic waves in neptunium monochalcogenides, Applied Acoustics, Vol. 72, No.10, 737-741, 2011.

[17] V. Bhalla, R. Kumar, C. Tripathy, D. Singh, Mechanical and Thermal properties of praseodymium monopnictides: An ultrasonic study, International Journal of Modern Physics B, Vol. 27, No.22, 1350116 (28 pages), 2013.

[18] D. Singh, S. Tripathi, D. K. Pandey, A. K. Gupta, D. K. Singh, J. Kumar, Ultrasonic wave propagation in semimetallic single crystals, Modern Physics Letter B, Vol. 25, No.31, 2377-2390, 2011.

[19] R. C. Hanson, Attenuation of high frequency elastic waves in LiF, Journal of Physics and Chemistry of Solids, Vol. 28, No.3, 475-483, 1967.

[20] D. Singh, R. R. Yadav, A. K. Tiwari, Ultrasonic attenuation in semiconductors, Indian Journal of Pure and Applied Physics, Vol. 40, No.12, 845-849, 2002.

[21] D. Singh, D.K. Pandey, P. K. Yadawa, Ultrasonic propagation in rare-earth monochalcogenides, Central European Journal of Physics, Vol.7, No.1, 198-205, 2009. 